\title{
Methodology for Testing the Influence of Buoyancy and Hydrodynamics on Swim Performance with and without a Wetsuit
}

\author{
Christopher R Harnish* \\ Department of Exercise Science, Shenandoah University, USA \\ *Corresponding author: Christopher R Harnish, PhD, Assistant Professor, Department of Exercise Science, Shenandoah University, Winchester, \\ VA 24088, USA
}

Submission: 眥 February 24, 2018; Published: 祭 April 27, 2018

\begin{abstract}
Purpose: The purpose of this pilot study was to develop test methodology that would allow for an estimation of the relative contributions of buoyancy and hydrodynamics on swim performance.

Methods: One trained swimmer completed three familiarization trials followed by eighteen randomized swim sessions where each condition no wetsuit (NS), low buoyancy wetsuit (LBW), high buoyancy wetsuit (HBW), NS plus pull buoy (NSB), and HBW matched to buoy buoyancy (HBW+) were tested four times each. Buoyancy for all conditions was measured via hydrostatic weighing system. All data are presented as means+SD and change scores $(95 \% \mathrm{CI})$.
\end{abstract}

Results: Coefficients of variations with each condition were about $2 \%$. D decreased by 2.29\% for LBW, $2.80 \%$ for HBW, $1.93 \%$ for NSB, and $1.96 \%$ for HBW+, which resulted in an increase in buoyancy lift force. LBW and HBW improved 800-yd swim times over NS -70.6 (-86.2, -55.0) sec, and -69.1 $(-84.0,-54.3) \mathrm{sec}$, respectively. Swim times for 100-yd were also similar between LBW, $-9.4(-10.7,-8.1)$ sec, and HBW, $-7.9(-11.4,-4.5)$ sec. Neither stroke rate nor total strokes differed between LBW and HBW, though both appeared significantly lower than NS. In HBW+ trials, the difference in 800yd and 100-yd times between NSB and NS was -25.2 $(-60.3,10.0)$ and -1.7 $(-19.6,-16.3)$, respectively, while HBW+ vs NSB 800-yd and 100-yd time differences were $-26.6(-28.3,-24.9)$ and $-5.7(18.9,7.5)$.

Conclusion: The outlined protocol can produce reliable results. These data support earlier assertions that buoyancy may reach a point of diminishing returns, and also indicate that wetsuit hydrodynamics play larger role in swim performance as velocity increases. The protocol outlined could aid in optimal wetsuit design without the need for advanced testing equipment.

Keywords: Wetsuits; Triathlon; Open water swim; Performance

\section{Introduction}

The growth of the sport of triathlon has witnessed tremendous growth in the past decade with approximately half a million members participating in 2015 [1,2] and has helped fuel the growth of several different types of open water swim competitions. The use of wetsuits in swim events, including triathlon, is typically permitted to protect against hypothermia, and is limited to a maximum neoprene thickness of $5 \mathrm{~mm}$. Modern high-performance wetsuits rely on variable thickness neoprene to optimize buoyancy in the water and minimize the affect on swim mechanics. In addition, they rely on smooth rubberized surfaces to reduce surface drag. Several studies [3-11] have demonstrated a significant reduction in swim times while using a wetsuit, while Tomikawa et al. [11] indicated that wetsuits may reduce oxygen cost during swims. Improved buoyancy is typically cited as the major contributing factor to faster swim times, and, to a lesser extent reduced surface drag.
However, some research [3-5] indicates that there is an upper limit to performance gains made by buoyancy, and that wetsuit design (e.g., buoyancy and drag) should be optimized based on athlete body composition and swim ability. It is not uncommon for wetsuit companies to offer many different wetsuit models with different neoprene thicknesses, including the maximum $5 \mathrm{~mm}$, followed by numerous marketing claims. However, it is unclear how these differences influence swim performance.

The purpose of this study was to develop and test a simplified model for describing the relative contributions wetsuit buoyancy and drag on swim performance as a proof of concept using several swim conditions with and without wetsuits. A second purpose was to compare two high-performance wetsuits of low and high buoyancy to that of a standard one-piece triathlon suit. We hypothesized that the higher buoyancy suit would result in faster swim times, but that wetsuits would also significantly improve swim drag. 


\section{Methods}

All testing procedures were conducted according to the policies and procedures of the Ferrum College IRB. One male volunteer completed nineteen swim trials during this study, including three familiarization trials. The subject was an experienced age group tri-athlete who had been competing for 7 years in road and offroad triathlons, and had a best race finish of $9^{\text {th }}$ overall at the 2016 USA Triathlon off-road national champions, as well as qualifying for several world championships. The subject was $1.75 \mathrm{~m}$ tall and weighed between 65.5 and $66.9 \mathrm{~kg}$ throughout the 8 -wk study period, and had a measured body density of $1.068245 \mathrm{~g} / \mathrm{m}^{3}$. The study took place during the subject's preparatory training period, and was not disrupted by any triathlon races in that time.

\section{Study design}

Following a 10-day familiarization period where the subject completed the test protocol three times wearing only a standard triathlon suit, a randomized cross-over design was used to assign Table 1: Summary of swim trial order and condition tested; NS: No Wetsuit; LBW: Lower Buoyancy; HBW: Higher Buoyancy Wetsuit; NSB: NS Plus Buoy; HBW+: HBW matched to B swim trials. Conditions tested included: no wetsuit (NS), a lower buoyancy XTerra Vengeance wetsuit (LBW), a higher buoyancy XTerra Vector Pro wetsuit (HBW), NS plus Finis pull buoy (NSB), and HBW matched to the buoyancy of NSB using $600 \mathrm{~g}$ of additional weight $(\mathrm{HBW}+)$. Weights were the same small precision weights used by the Monark Peak Bike and worn under the suit near the center of mass. In the first set of trials, each swim compared NS to LBW, HBW, and NSB; each condition was tested at least four times, twice as the first swim in a trial, and twice as the second swim in a trial. We then compared NSB with HBW+. The test conditions and order are summarized in Table 1. The wetsuits (XTerra Wetsuits, San Diego, CA) used in the study were tight fitting high performance racing suits with variable thickness neoprene and an external coating to surface drag; both wetsuits rely on $5 \mathrm{~mm}$ of neoprene in the lower extremities, $1.5 \mathrm{~mm}$ in the upper extremities, and $3 \mathrm{~mm}$ in the back, but the HBW use $5 \mathrm{~mm}$ vs $3 \mathrm{~mm}$ in the chest. Body density was measured for each condition was measured during the same week it was tested.

\begin{tabular}{|c|c|c|}
\hline Trial & Condition & LBW \\
\hline 1 & NS & NS \\
\hline 2 & NSB & NS \\
\hline 3 & LBW & HBW \\
\hline 4 & HBW & NS \\
\hline 5 & NS & NSB \\
\hline 6 & LBW & HBW \\
\hline 7 & NS & NS \\
\hline 8 & NS & NS \\
\hline 10 & HBW & LBW \\
\hline 11 & NSB & NSB \\
\hline 12 & NS & HBW+ \\
\hline 13 & NS & NSB \\
\hline 15 & NSB & NSB \\
\hline 16 & HBW & HBW+ \\
\hline
\end{tabular}

\section{Swim trials}

All swim trials were conducted in the same 25-yd pool and lane with a median (95\% CI) water temperature of 26.7 (26.1, 27.2), and at the same time of day (0900hrs). Each test session was separated by at least $72 \mathrm{hr}$. Following a standard 300 -yd warm-up at a perceived easy pace, the subject completed an 800-yd swim trial at their perceived 1600-yd race pace assisted by an audible head mounted Tempo Trainer Pro metronome (Finis, Inc. Livemore, CA) and set at $76 \mathrm{spm}$. Once complete, the subject rested in the water for 2 min before completing a maximal pace 100 -yd sprint. At the completion of the 100-yd swim, the subject exited the water and either removed or donned a wetsuit before re-entering the water and completing the $10 \mathrm{~min}$ rest period. A 100 -yd easy warm-up was then completed before another 800 -yd and 100 -yd sprint was completed; Table 2 summarizes the full swim trial. Timing was measured by the same technician using a standard stop watch, while Garmin 910 XT multisport watch (Garmin International, Inc., Olathe, KS) was used to measure stroke rate and other swim metrics.

Table 2: Detailed description of swim trial distances, intensities, and rest intervals. spm= Strokes/Minute

\begin{tabular}{|c|c|c|c|}
\hline Distance(Yd) & Relative Intensity & Rest Period(min) & Notes \\
\hline 300 & Easy & 1 & Warm-up \\
\hline 800 & 1600yd race pace & 2 & Metronome assisted rate of 76spm \\
\hline
\end{tabular}




\begin{tabular}{|c|c|c|c|}
\hline 100 & Maximal sprint & 10 & $\begin{array}{c}\text { Subject exited the water during rest period to either remove or } \\
\text { put on wetsuit }\end{array}$ \\
\hline 100 & Easy & 1 & Warm-up \\
\hline 800 & 1600 -yd race pace & 2 & Metronome assisted rate of 76 spm \\
\hline 100 & Maximal sprint & 1 & \\
\hline 100 & Easy & & \\
\hline
\end{tabular}

\section{Body density measurement}

Buoyancy was assessed as a function of body density [7], which was measured using an EXERTECH hydrostatic weighing system (Dresbach, MN) for LBW, HBW, HBW+, and NSB during each week it was tested, while NS buoyancy was measured in the same session each aforementioned condition. Buoyancy testing was identical to body density testing methodology described in the EXERTECH manual. All testing took place in the same pool used for the swim trials. During the wetsuit trials, a 12.4 belt was worn by the subject to help fully submerge. Residual lung volume was estimated based on height using the Exertech Manual, with the same value used for all calculations. Body density was calculated by dividing dry weight by body volume which was calculated using the following formula:

$$
\mathrm{V}_{\mathrm{B}}=\left[(\text { Dry-Wet }) / \mathrm{D}_{\mathrm{W}}\right]-\mathrm{RV}-0.1
$$

\section{Results}

Table 3: Summary of swim time and stroke count differences between no wetsuit and wetsuit or swim buoy conditions presented as differences scores $(95 \% \mathrm{CI})$.

\begin{tabular}{|c|c|c|c|c|}
\hline Trial Comparison & 800 Time Difference (sec) & 100 Time Difference (sec) & $\begin{array}{c}\text { 800 Stroke Difference } \\
\mathbf{( s p m )}\end{array}$ & $\begin{array}{c}\text { 100 Stroke Difference } \\
\mathbf{( s p m )}\end{array}$ \\
\hline NSB-NS & $-25.2(-60.3,10.0)$ & $-1.7(-19.6,16.3)$ & $-1(-13.7,11.7)$ & $1.0(-1,1)$ \\
\hline LBW-NS & $-70.6(-86.2,-55.0)$ & $-9.4(-10.7,-8.1)$ & $-28.5(-48.6,-8.4)$ & $-3.8(-5.8,-1.7)$ \\
\hline HBW-NS & $-69.1(-84.0,-54.3)$ & $-7.9(-11.4,-4.5)$ & $-23.8(-42.0,-5.5)$ & $-3.5(-8.4,1.4)$ \\
\hline HBW+-NSB & $-26.6(-28.3,-24.9)$ & $-5.7(-18.9,7.5)$ & $-7.5(-18.0,3.5)$ & $-3.2(-7.3,0.9)$ \\
\hline
\end{tabular}

Coefficients of variations for each condition were $2 \%$ or less. Table 3 summarizes all swim trial data. Compared to all other conditions, NS was significantly slower. Results indicate that $D_{B}$ decreased by $2.29 \%$ for LBW, $2.80 \%$ for HBW, $1.93 \%$ for NSB, and $1.96 \%$ for HBW+, which resulted in an increase in buoyancy lift force; relative buoyancy for HBW+ was matched to $0.8 \%$ of NSB. Compared to NS, swim times for 800-yd were -70.6 (-86.2, -55.0) sec, and $-69.1(-84.0,-54.3)$ sec for LBW and HBW, respectively. Swim times for 100-yd were also similar between LBW, -9.4 (-10.7, -8.1)sec, and HBW, -7.9 (-11.4, -4.5)sec. The total numbers of strokes were similar between LBW and HBW, though both were significantly lower than NS. In trials where HBW buoyancy was matched to that of NSB (HBW+), the difference in 800-yd and 100yd times between NSB and NS was $-25.2(-60.3,10.0)$ and -1.7 $(-19.6,-16.3)$, respectively, while HBW+ vs NSB 800-yd and 100yd time differences were $-26.6(-28.3,-24.9)$ and $-5.7(18.9,7.5)$, respectively. The total stroke count did not differ between HBW+ and NSB.

\section{Discussion}

The main purpose of this study was to develop and evaluate a relatively simple and cheap testing methodology for parsing out the relative contributions of increased buoyancy and improved
Where: $\mathrm{V}_{\mathrm{B}}=$ Body volume $(\mathrm{ml})$

$$
\begin{aligned}
& \text { BW=Dry weight }(\mathrm{kg}) \\
& \text { WW=Wet weight }(\mathrm{kg}) \\
& \mathrm{D}_{\mathrm{W}}=\text { Water Density }(\mathrm{g} / \mathrm{ml}) \\
& \mathrm{RV}=\text { Estimated residual lung volume }(\mathrm{ml})
\end{aligned}
$$

\section{Data analysis}

All data are presented as means $+\mathrm{SD}$, while comparison data are represented as difference scores from NS (95\% CI). Significance was defined as any difference greater than 0 with a $95 \% \mathrm{CI}$ that did not cross 0 . Due to the length of the study test period, coefficient of variations (CV) for swim times were calculated for each condition. hydrodynamics of high performance racing wetsuits. The secondary purpose was to compare the performance effects of LBW, HBW, and just high buoyancy (NSB and HBW+) on swimming. The major finding of the study was that it is possible to identify the impact of altering buoyancy and hydrodynamics can have on swim performance for different wetsuit designs. A second major performance finding of this study was that there appears to be an upper limit to improved performance by added buoyancy. Earlier studies [3-6] have demonstrated a 5-7\% improvement in swim times by using a wetsuit. Typically, improved buoyancy $[4,5,9]$, leading to improved body position, is cited for the main reason for this improvement, and, to a lesser extent hydrodynamics $[1,11]$. For example, Cordain \& Kopriva [6] showed a significant positive relationship between buoyancy and swim performance (i.e. faster times), and that subjects with higher body densities benefitted more than those with lower densities, suggesting that there may be a point of diminishing returns with increasing buoyancy. They provided further support for this when the added neoprene bands to the legs, which further increase buoyancy, but ultimately diminished performance. It is possible, however, that by only added more hydrostatic lift to the legs, they may have negatively impacted body position. Our current study, suggests that there may 
indeed be a maximum benefit to buoyancy, as well as evidence that current wetsuit technology improves swim performance through significant reductions in drag.

First, our subject demonstrated very high intra-test reliability, as evidenced by the low $\mathrm{CV}(<2 \%)$ between trials for a given condition. Based on the available data, we hypothesized that swim times would have been fastest during the HBW trials. In reality, there were no differences in swim time reductions between the LBW and HBW, with both improving 800-yd performance 7\%, while only the LBW consistently reduced 100-yd time. While the latter finding is probably attributed to the limited sample size, this suit is advertised to have the one of the lowest drag coefficients $\left(C_{d}\right)$ for commercial wetsuits. A lower $C_{d}$ would theoretically improve performance more as speeds increase. Beyond mere speculation, however, our data, taken in the context of earlier work $[1-6,10]$ suggest that wetsuits not only significantly improve swim times for all athletes, but may improve times for different reasons, depending on the ability level of the athlete. For instance, women tri-athletes like gain little additional benefit from a maximally thick wetsuit, while very lean, and perhaps muscular athletes likely would. Athletes with a high swimming ability likely benefit most from a suit minimize $\mathrm{C}_{\mathrm{d}}$.

A secondary objective of the study was to test whether we could reliably match buoyancy of our HBW to that of a standard small pull buoy, thereby investigating the contribution of reduced drag on swimming. We did this by using common precision weights used during Wingate testing on the Monark bike. These weights were secured near the center of mass and provided a balanced downward force without altering overall body position and reducing buoyancy. In this condition, we matched measured body density to within $0.03 \%$ of the buoy. From this condition, swim times were only about $26 \mathrm{sec}$ faster than NS. If we consider that the reduced buoyancy resulted in a portion of the loss of velocity, we can estimate that 30 $40 \%$ of the improved swim times could be related reduction in drag, while buoyancy accounts for roughly $60-70 \%$ of the gains in speed. It is important to qualify this statement, however, by remembering that improved buoyancy results in an improved body position in the water. Because $C_{d}$ is a function of both form drag and surface drag [8], it is impossible to determine here the extent that surface, or skin, drag played, but research by Mollendorf et al. [9] suggests that at speeds about $1 \mathrm{~m} / \mathrm{s}$, frontal pressure drag is greatest, with skin drag playing an increasingly greater role as speed increases. Thus, improved body position itself would reduce pressure drag considerably, while the reduced skin drag would reduce drag more for faster swimmers. Research by Chatard \& Wilson [2] indicates that full body drag reducing suits alone can significantly improve performance.

One lesser finding of this study was the impact of wetsuits on stroke rate and total strokes. Prior research [4,9] has reported that stroke rate increased proportionally with the improvement in performance; i.e., the wetsuit improved times, the more stroke rate increased. In order to better control our intra-trial reliability, our subject used an audible metronome, thus stroke rate did not vary significantly. However, in both HBW and LBW conditions the total number of strokes over $800 \mathrm{yd}$ was nearly $7 \%$ lower than NS. This would indicate that the distance covered per stroke is significantly greater when wearing a wetsuit. Data published by Tomikawa et al. [11] suggest that wearing a wetsuit reduces sub-maximal oxygen cost. It seems logical that regardless of stroke rate, if fewer strokes are taken over a given distance, then energy cost would also be reduced.

In conclusion, our study is a proof of concept indicating that relatively simple testing methods can be employed to study the impact of buoyancy and lift on swimming performance with and without a wetsuit. Our data support earlier research showing that wearing a high-performance wetsuit significantly improves swim times, and may actually reduce energy cost [12]. In is incumbent upon future researchers to expand our research to include a larger subject pool to further elucidate the role buoyancy and drag play on swim performance. Practical applications for this study are that athletes and coaches should consider their swimming ability and body composition when choosing a wetsuit; in general, the suit with the highest buoyancy is not always the fastest. Highly skilled swimmers would likely benefit from a wet suit with the lowest skin drag. Finally, our data do not support common triathlon federation rules on neoprene thickness regarding performance enhancement.

\section{Acknowledgement}

This research was made possible through a Ferrum College new faculty grant and XTerra Wetsuits. I also wish to thank Kaitlyn Yachem, Ashley McAllister, and Carmen Waslicki for their help in data collection.

\section{References}

1. USA triathlon 2015 membership report.

2. Bentley DJ, Cox GR, Green D, Laursen PB (2008) Maximising performance in triathlon: Applied physiological and nutritional aspects of elite and non-elite competitions. J Sci Med Sport 11(4): 407- 416.

3. Chatard JC, Wilson B (2008) Effect of fast skin suits on performance, drag, and energy cost of swimming. Med Sci Sports Exerc 40(6): 1-7.

4. Chatard JC, Millet G (1996) Effects of wetsuit use in swimming event: practical recommendations. Sports Med 22(2): 70-75.

5. Chatard JC, Senegas X, Selles M, Dreanot P, Geyssant A (1995) Wet suit effect: a comparison between competitive swimmers and triathletes. Med Sci Sports Exerc 27(4): 580-586.

6. Cordain L, Kopriva R (1991) Wetsuits, body density and swimming performance. Br J Sports Med 25(1): 31-33.

7. de Lucas RD, Balikian P, Neiva CM, Greco CC, Denadai BS (2000) The effects of wet suits on physiological and biomechanical indices during swimming. J Sci Med Sport 3(1): 1-8.

8. Katch F, Michael E, Horvath SM (1967) Estimation of body volume by underwater weighing: description of a simple method. J Appl Physiol 23(5): 811-813.

9. Mollendorf JC, Termin AC, Oppenheim E, Pendergast DR (2004) Effect of swim suit design on passive drag. Med Sci Sports Exerc 36(6): 10291035 . 
10. Parsons L, Day SJ (1986) Do wet suits affect swimming speed? Brit Sports Med 20(3): 129-131.

11. Tomikawa M, Shimoyama Y, Nomura T (2008) Factors related to the advantageous effects of wearing a wetsuit during swimming at different submaximal velocity in triathlete. J Sci Med in Sport 11(4): 417-423.
12.Toussaint HM, Bruinink L, Coster R, De Looze M, Van Rossem B, et al. (1989) Effect of a triathlon wetsuit on drag during swimming. Med Sci Sports Exerc 21(3): 325-328.

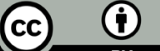

Creative Commons Attribution 4.0 International License

For possible submissions Click Here



